\title{
Prevention of Data Theft Attacks in Infrastructure as a service Cloud through Trusted Computing
}

\author{
Gogineni Krishna Chaitanya, Amarendra.K, Shaik.Aslam, Uppuluri Lakshmi Soundharya, \\ Vailipalli Saikushwanth
}

\begin{abstract}
Dispensed computing is a developmental model that permits cloud consumer to name for assets with on-request management and use the assets utilizing pay in step with use version. Due to this pliability, the dispensed computing implementing colossal safety dangers. Consistent with the pc security Alliance, an insider chance is a noteworthy chance in distributed computing situation. This threat quite influences the data trustworthiness and classification of cloud client applications, administrations, or statistics. In this paper, we generally have a tendency to specializing in corporation reliable attacks on consumer virtual machines and anticipated a structure that upholds facts respectability and privateness, littlest TPM activities, and decreased and irrefutable reliable figuring base. Our exploratory consequences show the us of the united states that custom remember-visor hyper-visor playacting brilliant and it will prevent and word the business enterprise legitimate assaults in cloud surroundings.
\end{abstract}

Catchphrases: Insider attack, hypervisor, confided in figuring, counteractive action

\section{ADVENT}

Dispensed computing is companion in Nursing rising and promising worldview that gives cloud customers to shop their belief and processing assets on interest with pay-in step with-use version. A in addition issue of a distributed computing makes thrive and further triple-crown than the Grid processing like automobile-scaling, low fee and multi inhabitance. A extraordinary reception of cloud administrations winds up in companion in Nursing corporate respectable assault [18], that extensively harms the information class and trustworthiness in cloud air. Cloud agency will make certain getting to know respectability and kind by regularly setting away cloud customer records in encoded company and interpret the ones at the cloud diploma. This system finally finally ends up in high way cost [19] and it is no longer affordable in cloud tiers. The homomorphic coding plans [15], plays calculations on encoded mastering at that aspect produces result as companion in Nursing re-appropriated. The cryptography task fits aftereffects of activities completed on unique

Revised Manuscript Received on April 12, 2019.

Gogineni Krishna Chaitanya, Department of Computer Science Engineering, Koneru Lakshmaiah EducationFoundation, Vaddeswaram, Guntur, AP, India. (E-mail: gkc_chaitu@kluniversity.in)

Amarendra.K, Department of Computer Science Engineering, Koneru Lakshmaiah EducationFoundation, Vaddeswaram, Guntur, A.P, India.

Shaik.Aslam, Department of Computer Science Engineering, Koneru Lakshmaiah EducationFoundation, Vaddeswaram, Guntur, A.P, India

Uppuluri Lakshmi Soundharya, Department of Computer Science \& Engineering, SRM University. Chennai, T.N, India.

Vailipalli Saikushwanth, Department of Computer Science \& Engineering, Koneru Lakshmaiah Education Foundation, Vaddeswaram, Guntur, A.P, India mastering. These methods produces first-rate measure of overheads in appearing task and in affordable absolutely crude sports are upheld in cloud air. Any mission finished in IaaS cloud should be apparent to cloud patron, it is a hearty interest in cloud foundation.

\section{RELATED ARTWORK}

Confided in Computing based completely methodologies Khan et al. [7], prepared a way to attain the customer records calculation classification and trustworthiness in IaaS cloud. The concept of this approach offers with dependable digital machine display (TVMM) and some distance flung affirmation, interior which reliable gathering verification the extent abuse PCR hash price. The cloud management company check in and verifies the North Carolina with a long way flung reliable collecting abuse TPM far off verification property. To such an quantity that, dependable collecting acclimated set up a be given as genuine with amongst cloud consumer and cloud issuer.

S Butt et al. [8] organized Cloudvisor, it maintains the valid area from obtrusive information secrecy and trustworthiness of CC VMs misuse settled virtualization notion. In Xen based for the most element shape, space administrator(Dom0) answerable for consumer VMs uprightness and privateness. Each unmarried advantaged mission are taken consideration through every settled and empty metalhypervisor that log jams the purchaser applications and that $\mathrm{i} / \mathrm{O}$ interfaces. Therefore, settled virtualization concept brings approximately large overheads in cloud framework.

Krautheim et al. [9] investigated digital putting on a cloud basis to make certain affirmation on records calculation and patron learning. TVEM underpins an Intel dependable eXecutionEnvironment (TXT) and virtualization innovation that permits the immediate $\mathrm{O} / \mathrm{i}$ am getting to. Inside the TXT, decided on errands are dispensed to useful resource company and studying owner within the direction of this arranged philosophy. TVEM offers capability to information proprietor to endure witness to the digital placing for secrecy and honesty.

Land organized thru Garfinkel et al. [10], offers a secluded execution putting in shut box and it's miles a universally beneficial framework feature through manner of component within the direction of a solitary degree. Land makes the correspondence as clean in hub correspondence 
with circulated putting misuse TVMM. The TVMM guarantees capacity to the board VM for asset association and designation of capability. TVMM offers far flung gatherings to go through witness to the customer VM in shut discipline execution putting to make sure degree and getting to know trustworthiness and confidentiality. This technique counteracts chiefs and ring 0 desired clients from adjusting or attending to purchaser VMs misuse segregated execution putting that ensures statistics uprightness and privateness.

A method prepared through Dewan et al. [11] offers a gatekeeper to the sensitive learning of client software going for walks in the path of a virtual execution setting. All through this device, customer VM may additionally contains malware for you to impacts the cloud level. Light-weight hypervisor used in this method that ensures run time firstrate grained programming package memory security and it places software sensitive learning in the predetermined ensured memory location. At that factor, it registers with client software and getting to of secured reminiscence vicinity limited with the aid of the use of hypervisor or VMM abuse affirmation. This approach gives a storage element to hypervisor or VMM to defend or save you analyzing spill from application stockpiling to root packs and malware.

Self-company Clouds [12], limits the hoop zero advantaged frame space in Xen placing from analyze the patron VM substance and calculation expertise.SSC isolates the particular frame place undertakings into framework wide location and a for each patron frame area. Upheld the saved rights, consumer will carry out pastime all by myself VMs.

Murray et al.[13], arranged a disaggregation property that produces a long way off validation more pregnant and reduce again the TCB estimate. Creators broke down Xen form and they prepared trade in Xen based totally usually shape in the organized approach. The region constructing strategy enthusiastic from frame vicinity to super area known as area B.

\section{HASSLE ANNOUNCEMENT}

In this region, we gift the threat fashions in which an insider can manage purchaser VM's within the NC of Cloud diploma. Here, we anticipated that CSP is vindictive and CU is not having any safety imperatives to get to their cloud sources. The version portrayed in areas in cloud framework.

\subsection{Assaults inside the local device}

We anticipate that the endowment OS with malignant company respectable at cloud diploma. With the forestall aim that, it it seems that certainly expresses that component and client mode isn't modified. With the prevent intention that, A agency professional will bargain VM's, those are walking on controller of purpose bunch. As an example, A company professional will modify goal VM element and that they'll dispatch VM with pernicious goal without any consents from VM owner (purchaser). It brings approximately touchy information damage of allocated storage of numerous VM and goal VM. In regards of this attack layout, VM's the ones are taking walks on $\mathrm{NC}$ are in decent hazard from insiders..

3.2 Assaults within the cloud supervisor
Assaults inside the cloud supervisor CSP includes ring zero advantages to get to any substance of cloud clients and physical assets facilitated at cloud facts recognition. To dispatch employer valid attack on belongings, company decent receives a memory sell off of objective VM. Belly muscle initio pernicious employer expert has no arrangement regarding accreditations hold in sell off of VM bit photograph. To get a catchword from piece, accomplice transgressor or corporate first rate simplest devises a route on got little little bit of VM. The detail photo sifted abuse strings order, it simply appears at sell off and returns there strings with call of slogan. Whilst organisation real acquires qualifications from a part of VM, the resulting are everyday troubles:

- A CSP gets to visitor OS substance via misuse their advantages. With aftereffect of this Cloud patron might conceivably lose their information secrecy and honesty. As previously stated earlier than, CSP will spare, reestablish, reboot, and closedown any visitor programming framework.

- In [1] incontestable fluctuated attack instances and individuals make superb risks in allotted computing digital environment.

- A pernicious corporate reliable or vindictive CSP will revision or rupture facts upon joined with contenders of the patron agency. Assailants (insiders) in the business enterprise have first rate danger to facts belongings because of they may be delicate concerning inward shape.

- Malicious company professional cannot get to the hypervisor besides they may get to capacity machine and system I/O. With this hazard CSP will play out any challenge without any consent from owner of domain or digital machine.

\subsection{Obtaining personal keys victimisation memory snapshots}

The essential target of second assault is to gather theprivate key of private-open key join inside the cloud setting. This assault situation shows anyway a key acquired from the Apache net server. The key's utilized for making or setting up a safe channel with customers. As appeared inside the prior assault, the individual key's keep in memory dump inside the style of plain content arrangement. Here, RSA key's assortment either 1024 or 2048 bits.

\$ xm dump-core 2 -L sekhardomu.dump

Dumping core of domain: 2 ...

$\$$ rsakeyfindsekhardomu.dump

found private key at 1b061de8

version $=00$ modulus $=00 d 066 f 89 d$ e2 be $4 a 2 b 6 d$ be $9 f \mathrm{de} 46 \mathrm{db} 5 \mathrm{a} \ldots$

publicExponent $=010001$

privateExponent $=\ldots$

prime $1=\ldots$

prime $2=\ldots$

To dispatch this attack, a malevolent commercial enterprise legitimate receives a memory unload of client VM, as prior attack. Currently, the commercial enterprise 
proper having keys within the reminiscence sell off anyway reminiscence unload duration is least of many MB's. During this attack we generally tend to applied the indistinguishable approach to get the non-open keys from the memory dump as virus boot assault. The crypto coherent keys are draw close on in the memory sell off are in perceived arrangement i.E., maximum abuse PKCSnumber 1 that speaks to the keys in ASN.1 article institution. To such an quantity that, ASN.1 having famous shape of RSA key in the memory promote off. The rsakeyfind system seek the memory promote off to separate the RSA enters in well-known item structure. The consequent course demonstrates the assault order succession on UNIX framework degree.

\section{PROPOSED FRAMEWORK \& RESULTS}

On this location, we have a propensity to sum matters up clarified concerning arranged framework an excellent manner to prevent achievable corporate official attacks in cloud putting from metal detail and CSP sees.

\subsection{Technique}

Our device uses machine based totally normally protection alluded to as TPM (trusted Platform Module), this is prepared with the resource of pool organization alluded to as dependable Computing Base (TCB). The most motivation inside the again of TPM is to defend sensitive statistics from out of doors taking however as from inward gatherings in cell phone, laptop and PDA's. In the arranged framework, differed TPM administrations are wont to affirm facts of client VM. Our shape takes a shot at Infrastructure as a carrier (IaaS) model, on this way we are able to in significant remember VM and its related assets in cloud surroundings. As clarified in advance than, we typically tend to applied eucalyptus cloud computer code for actualizing IaaS based totally for the most detail cloud and fig one in a nutshell demonstrates besides organized framework works in cloud. Eucalyptus is open deliver cloud pc code and it is API-suited with EC2.We implemented eucalyptus fashion for actualizing cloud foundation, that may be a answer for enterprise Amazon EC2.Node Controller (NC) need to introduce on TrustVisor hypervisor and VM's are putting in on North Carolina (exposed metal hypervisor).

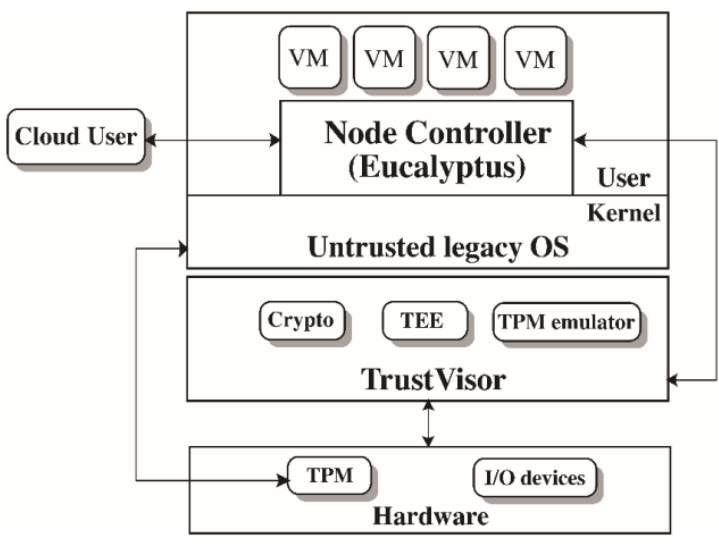

Fig. 1: Proposed framework

The NC's are sign up with the the front end hub for UI. The CU demand NC with open key $\%$ and nonce esteem for VM execution. The NC tests the open key Pkcand nonce then advances the open key and nonce (that's gotten from the CU)to the TrustVisor module through TPM. TrustVisor checks the p.C and after that understand with the nonce to $\mathrm{CU}$ for non-public key percentage. The pkcof $\mathrm{CU}$ gave to TrustVisor to decoding of VM and NC unscramble VM with $\%$. Currently, CU can get to the VM and every VM contain $\mu$ TPM for verifying the substance by using manner of its personal. The $\mu$ TPM is a chunk of the TrustVisor on NC $\mathrm{CPU}$, with the forestall intention that it takes out the terrible execution sway on successive usage of dynamic basis of receive as true with [2] (vTPM[3], Flicker[17]). The TrustVisor is an exposed metal hypervisor, which offers a disengaged execution circumstance and "miniaturized scale" TPM concept for verifying VM substance thru secure sensitive Code Block (SSCB).At ease sensitive code squares are formally referred to as Piece of application common sense(pal).

\subsection{Comfy past due VM dispatch}

On this section we will be inclined to portray at ease VM dispatch approach in cloud framework abuse TPM far flung verification on TrustVisor [2] and phase 5.Three depicts concerning anyway software are useless in disconnected placing on pinnacle of the TrustVisor. This tool accomplishes the statistics honesty of customer packages which can be strolling on trusty hypervisor.An outer trusty outsider or supporter gets a TPM-created authentication that has set of PCR traits and following data that should be stretched out to skip on the consequent statistics [2]:

- SKINIT steering (AMD) implemented in bootstrap for the execution of TrustVisor abuse dynamic root trust.

- next, TrustVisor gets the management of dynamic basis of trust.

- one in all of the PCRs incorporates cryptanalytic hash (estimation) of TrustVisor

- TrustVisor creates the personality key abuse contemporary TPM AIK for $\mu$ TPM.

A TrustVisor affirmation accommodates set of yields of HV_quote hobby together with alternative untrusted facts to the sponsor making sense out of the $\mu$ PCRs. The trusty celebration or supporter must confide within the TrustVisor abuse TPM authentication document. Count on TrustVisor is untrusted, at that point whole cloud placing will don't forget as untrusted. To such an volume that, no trust putting can be made with untrusted TrustVisor [2].

\subsection{Implementation}

The protrusile state-of-the-art Hypervisor Framework has been applied to make the TrustVisor hypervisor collectively with the middle modules: cryptography duties, TEE and TPM human, includes TPM library paintings to shape a blanketed correspondence with TPM tool. The made hypervisor has been set inside the cloud server grub passage to border a dedication of hypervisor. A great way to, make certain the trust decency of cloud diploma a eliminated authentication concept, we generally tend to implemented maximum properly-loved and extensive applied method alluded to as Integrity movement plan (IMA)[4]. Some 
distance off validation uses IMA, it certainly works upheld twofold verification idea. When designing IMA, it figures and broadens the hashes all matters taken into consideration at the same time as boot method into their outstanding PCRs. To make certain the far off validation with protection moderating of North Carolina, we generally generally tend to implemented Attestation identification Key(AIK) for gesture based totally conversation hashes of PCRs although playacting declaration challenge. We typically tend to implemented TPM human for correspondence with TPM system exploitation TPM driving force.

As referenced earlier than, we generally tend to utilized open supply cloud code alluded to as eucalyptus to decide anIaaS character cloud for our sorting out. Eucalyptus [5] accommodates very a good buy sketched out components like North Carolina, CC, walrus, SC and Cluster Controller(CSC), the ones gives efficient correspondence amongst belongings exploitation internet-administration. Eucalyptus is companion diploma EC2 API-tremendous and this is a solution for commercial enterprise Amazon EC2 cloud foundation. Eucalyptus underpins libvirt hypervisor, it accommodates maximum well-enjoyed hypervisors Xen and KVM hypervisors. The eucalyptus factors are very a good deal delineated with net-management basically based totally interfaces and individuals elements are created exploitation bizarre country and popular bundles like Axis2, Apache, and economic organization [6]. Our organized plan uses the ones additives to demonstrate that our idea is relaxed from commercial company real assaults.

The North Carolina is that the focal difficulty of our organized eucalyptus cloud component work, whereCC will dispatch and execute VMs. The organized shape legal on HP first magnificence notebook 8540 with setup of Intel i5 processor, 8GB RAM, 500HDD and Ubuntu fourteen.04 as quite quite a number OS. The Eucalyptus cloud code carried out for actualizing the individual cloud that has partner diploma framework for propelling VM's. The check results reveal that prepared shape has large capacity to downsize the TCB minimisation and lots much less over heads even though talk with TPM machine thru the host package deal.

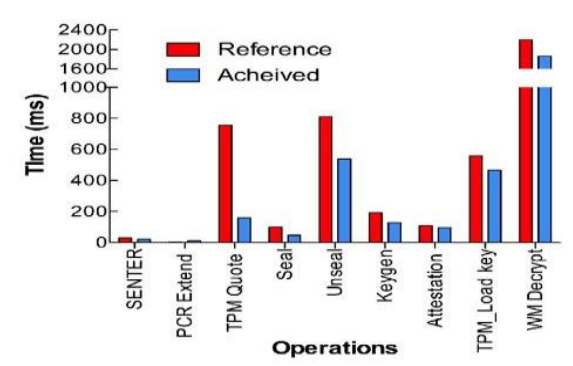

Fig. 2: Performance evaluation

The SENTER steering takes twenty. $5 \mathrm{~ms}$ for the graduation of comfortable boot collectively with the TrustVisor hypervisor boot method. The PCR make bigger is implemented to cite specific PCR charge and it took ten.68ms. The TPM quote for measure the PCR esteems with hash esteems are decided and supplanted with new hash assessment and this activity took $357.68 \mathrm{~ms}$. Along those strains it shows North American kingdom that radiance fundamentally based surroundings units apart surprisingly lengthy attempt to counter for the TPM quote. The seal and open up pastime takes $40537.87 \mathrm{~ms}$, when located by numerous hypervisor execution in every venture TrustVisor has incredible potential to lessen the overheads in open up hobby. The a ways flung verification took $100.3 \mathrm{~ms}$ for confiding inside the degree misuse the PCR esteems with technological know-how strategies the ones we generally tend to referenced earlier than regions. The effects display North American state that TrustVisor has nice ability to cut back the overheads throughout the TPM operations.

\begin{tabular}{|l|l|l|l|l|}
\hline & Extend & Seal & Unseal & Quote \\
\hline $\begin{array}{l}\text { Native } \\
\text { Linux }\end{array}$ & 24066 & 358102 & 1008654 & 815654 \\
\hline TrustVisor & 533 & 11.7 & 12.6 & 21000 \\
\hline
\end{tabular}

\begin{tabular}{|l|l|l|l|l|}
\hline & \multicolumn{2}{|c|}{ HMAC } & \multicolumn{2}{c|}{ Sign } \\
\hline & Avg & Stdev & Avg & Stdev \\
\hline Flicker & 62.644 & 0.181 & 67.461 & 0.008 \\
\hline TrustVisor & 0.051 & 0.003 & 5.012 & 0.018 \\
\hline
\end{tabular}

Table 1: HMAC and Basic operations on TrustVisor(ms) [2]

The desk one delineates the exhibition of TrustVisor on cloud placing. HMAC is carried out to get the hash condensation of programming framework final in PCR registers. Those registers are implemented for trustworthiness test of patron virtual tool substance. The avg and distinction is decided on TrustVisor and Flicker. Glimmer takes sixty .644ms any vicinity as TrustVisor takes zero. $059 \mathrm{~ms}$, it demonstrates the exhibition and ward nature of execution of cloud components on hub controller. At some stage in this device, we will ascertain the beginning deviation of each thing are: $0.181 \mathrm{~ms}$ severally. It use the possibility of TrustVisor in cloud putting. Decided this could paintings with elite in execution reason for view properly as in usage of cloud additives.

\section{TRUSTED COMPUTING BASE}

\begin{tabular}{llll}
\hline Hypervisor & Hypapp & XMHF core & Total \\
\hline TrustVisor & 3939 & 6018 & 9957 \\
LockDown & 9391 & 6018 & 15409 \\
XTRec & 3500 & 6018 & 9518 \\
SecVisor & 2200 & 6018 & 8218 \\
\hline
\end{tabular}

Table 2: LOC comparison with other hypervisors [23]

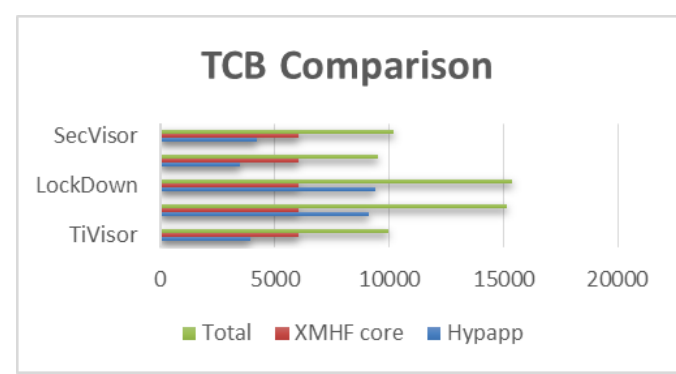

Fig. 3: TCB comparison

Published By: Blue Eyes Intelligence Engineering \& Sciences Publication 


\section{REMOTE ATTESTATION PERFORMANCE}

The number one problem with the prior tool (Park at al., 2015 ) is that consumer can incidentally or intermittently solidifying for extra or less for notably $550 \mathrm{~ms}$ inside the remote validation level. This trouble harms the cloud customer at the same time as playing out the remote validation with cloud server. The hassle is a proper away give up end result of horrific TPM sports, for example, TPM_Quote2. To such an extent that, we supplant huge duties with insignificant sports activities within the product cryptographic sports for confirmation convention. To break down the exhibition of a ways flung validation, TrustVisor makes use of a 1024 piece session key, this is made or created in boot device. The under table shows, the presentation of various hypervisors. The neighborhood Linux takes 518.1ms, TGVisor takes $172.1 \mathrm{~ms}$, TrustVisor takes $288.3 \mathrm{~ms}$, SecVisor takes $240.5 \mathrm{~ms}$, Lockdown takes $298.3 \mathrm{~ms}$ and Proposed tool with TrustVisor takes $169.8 \mathrm{~ms}$. Ultimately, the TrustVisor sets apart much less effort for far off confirmation for the a long way off authentication.

\begin{tabular}{|c|c|c|c|c|l|}
\hline $\begin{array}{c}\text { Native } \\
\text { Linux }\end{array}$ & TGVisor & $\begin{array}{c}\text { Cloud } \\
\text { Visor }\end{array}$ & $\begin{array}{c}\text { Sec } \\
\text { Visor }\end{array}$ & $\begin{array}{c}\text { Lockd } \\
\text { own }\end{array}$ & $\begin{array}{l}\text { Propo } \\
\text { sed }\end{array}$ \\
\hline 518.1 & 172.1 & 288.3 & 240.5 & 298.3 & 169.8 \\
\hline
\end{tabular}

Table 3 : Performance evolution of Remote attestation [23]

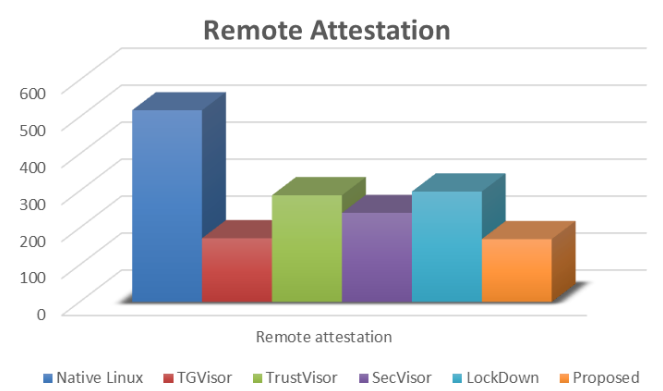

Fig. 4: Remote attestation evolution

As appeared inside the fig 4, the organized structure convention in the faraway verification beats the earlier hypervisors via more than one. 88 instances due to the arranged machine uses mild-weight TPM activities like TPM_PCR_Extend and TPM_PCR_Extend to test or verify the trustworthiness of the inspiration any area VM to be useless or being execution. The TPM_PCR_Read takes seven.2ms and TPM_PCR_Extend takes $8.2 \mathrm{~ms}$ to refresh the PCR sign on. Such the TPM responsibilities takes much less quantity overheads despite the fact that playacting the remote authentication with far flung collecting.

\section{PREVENT}

This research had 2 reasonable examination stages. Introductory work targeted on displaying anyway modernday-day virtualization layer programming framework is not possible at counteracting attacks starting from a vindictive corporate professional. The thinking of and attempting out a totally one among a kind cloud plan that can prevent the noxious enterprise reliable assaults previously stated in our art work. Inside the path of this diploma, we generally tend to applied accomplice in Nursing eucalyptus programming framework for virtualization and changed its memory the board gadgets to demonstrate the hyper-visor in to the secure from the employer expert attacks within the form. This adjustment is abrogating to guarantee a similarly dependable shape even though diminishing its reliable figuring base.

\section{REFERENCES}

1. Halderman, J.A., Schoen, S.D., Heninger, N., Clarkson, W., Paul, W., Calandrino, J.A., Feldman, A.J., Appelbaum, J. Moreover, Felten, E.W., 2009. In case we maintain in mind: bloodless-boot attacks on encryption keys. Correspondences of the ACM, fifty two(5), pp.91-98.

2. McCune, J.M., Li, Y., Qu, N., Zhou, Z., Datta, A., Gligor, V. Furthermore, Perrig, A., 2010, can also. TrustVisor: inexperienced TCB lower and authentication. In 2010 IEEE Symposium on protection and privacy (pp. 143-158). IEEE.

3. S. Berger, R.Caceres, ok.A.Goldman, R.Perez, and R.Sailer, and L. Van Doorn, vTPM: Vitalizing confided in degree module. In Proc. USENIX safety Symposium.2006.

4. Sailer, Reiner, et al. "Plan and Implementation of a TCGprimarily based Integrity size structure." USENIX protection Symposium. Vol. 13. 2004.

5. Nurmi, Daniel, wealthy Wolski, Chris Grzegorczyk, GrazianoObertelli, Sunil Soman, Lamia Youseff, and DmitriiZagorodnov. "The eucalyptus open-deliver dispensed computing framework." In Cluster Computing and the Grid, 2009. CCGRID'09. Ninth IEEE/ACM international Symposium on, pp. 124-131. IEEE, 2009.

6. I. Khan; Z. Anwar; B. Bordbar; E. Ritter; H. U. Rehman, "A Protocol for stopping Insider attacks in Untrusted Infrastructure-as-a-service Clouds.," in IEEE Transactions on Cloud Computing ,vol.PP, no.99, pp.1-1.Doi: 10.1109/TCC.2016.2560161

7. Khan, I., Rehman, H.U. What's more, Anwar, Z., 2011, July. Plan and sending of a believed eucalyptus cloud. In Cloud Computing (CLOUD), 2011 IEEE worldwide convention on (pp. 380-387). IEEE.

8. Zhang, Fengzhe, et al. "CloudVisor: retrofitting coverage of virtual machines in multi-inhabitant cloud with settled virtualization." court cases of the Twenty-1/3 ACM Symposium on working structures concepts. ACM, 2011.

9. Krautheim, F. John, Dhananjay S. Phatak, and Alan T. Sherman. "presenting the confided in digital situation module: any other system for organising agree with in disbursed computing." international convention on recall and sincere Computing. Springer Berlin Heidelberg, 2010.

10. Garfinkel, Tal, et al. "Land: A virtual tool-primarily based degree for confided in registering." ACM SIGOPS working structures assessment. Vol. 37. No. 5. ACM, 2003.

11. Dewan, Prashant, et al. "A hypervisor-primarily based framework for securing programming runtime reminiscence and industrious stockpiling." proceedings of the 2008 Spring replica multiconference. Society for computer Simulation worldwide, 2008.

12. Butt, Shakeel, et al. "Self-management distributed computing." court docket cases of the 2012 ACM assembly on computer and correspondences safety. ACM, 2012.

13. Murray, Derek Gordon, Grzegorz Milos, and Steven Hand. "enhancing Xen protection thru disaggregation." lawsuits of the fourth ACM SIGPLAN/SIGOPS global accumulating on virtual execution conditions. ACM, 2008. 
14. Tysowski, Piotr k., and M. Anwarul Hasan. "move breed great and re-encryption-based totally key management for secure and bendy portable packages in mists." IEEE Transactions on Cloud Computing 1.2 (2013): 172-186.

15. Naehrig, Michael, Kristin Lauter, and Vinod Vaikuntanathan. "Can homomorphic encryption be useful?." court cases of the $1 /$ three ACM workshop on Cloud processing protection workshop. ACM, 2011.

16. Lei, Xinyu, et al. "Redistributing large grid reversal calculation to an open cloud." IEEE Transactions on allotted computing 1.1 (2013): 1-1.

17. McCune, Jonathan M., et al. "Glimmer: An execution framework for TCB minimization." ACM SIGOPS operating systems overview. Vol. Forty two. No. Four. ACM, 2008.

18. Kroes, Neelie. "putting in vicinity the european cloud enterprise." global financial forum, Davos, Switzerland, twenty sixth Jan. 2012.

19. Kamara, Seny, and Kristin Lauter. "Cryptographic disbursed garage." global convention on economic Cryptography and records safety. Springer Berlin Heidelberg, 2010.

20. Parno, Bryan. "The confided in level module (TPM) and fixed stockpiling." TPM Documentation. June twenty first (2007).

21. Coker, George, et al. "requirements of some distance flung confirmation." worldwide magazine of information safety 10.2 (2011): sixty three-81.

22. Park, Sungjin, et al. "A minor hypervisor-based completely confided in geolocation form with limited TPM sports." magazine of systems and software program 122 (2016): 202214.

23. Rocha, Francisco, and Miguel Correia. "Lucy in the sky without precious stones: Stealing mystery statistics within the cloud." reliable structures and Networks Workshops (DSNW), 2011 IEEE/IFIP forty first worldwide conference on. IEEE, 2011. 